Research Article

\title{
Characterization of Medication Trends for Chronic Kidney Disease: Mineral and Bone Disorder Treatment Using Electronic Health Record-Based Common Data Model
}

\author{
Sungdam Han $\mathbb{D}^{1},{ }^{1}$ Minkook Son $\mathbb{D}^{2},{ }^{2}$ Byungjin Choi $\mathbb{D}^{3},{ }^{3}$ ChulHyoung Park $\mathbb{D}^{\circ},{ }^{3}$ \\ Dong Ho Shin $\mathbb{D}^{4}{ }^{4}$ Jong Hwan Jung $\mathbb{D}^{5}{ }^{5}$ Min-Jeong Lee $\mathbb{D}^{1},{ }^{1}$ Gyu-Tae Shin $\mathbb{D}^{1}{ }^{1}$ \\ Heungsoo Kim $\mathbb{D}^{1},{ }^{1}$ Rae Woong Park $\mathbb{D}^{3,}, 6$ and Inwhee Park $\mathbb{D}^{1}$ \\ ${ }^{1}$ Department of Nephrology, Ajou University School of Medicine, 164, Worldcup-ro, Yeongtong-gu, Suwon, \\ Gyeonggi-do 16499, Republic of Korea \\ ${ }^{2}$ Department of Biomedical Science and Engineering, Gwangju Institute of Science and Technology, 123, Cheomdangwagi-ro, Buk- \\ gu, Gwangju 61005, Republic of Korea \\ ${ }^{3}$ Department of Biomedical Informatics, Ajou University School of Medicine, 164, Worldcup-ro, Yeongtong-gu, Suwon, \\ Gyeonggi-do 16499, Republic of Korea \\ ${ }^{4}$ Department of Internal Medicine, College of Medicine, Hallym University, Kandong Sacred Heart Hospital, 150, Seongan-ro, \\ Gangdong-gu, Seoul 05355, Republic of Korea \\ ${ }^{5}$ Division of Nephrology, Department of Internal Medicine, Wonkwang University School of Medicine and Hospital, 895, Muwang- \\ ro, Iksan, Jeollabuk-do 54538, Republic of Korea \\ ${ }^{6}$ Department of Biomedical Sciences, Ajou University Graduate School of Medicine, 164, Worldcup-ro, Yeongtong-gu, Suwon, \\ Gyeonggi-do 16499, Republic of Korea
}

Correspondence should be addressed to Rae Woong Park; veritas@ajou.ac.kr and Inwhee Park; inwhee@aumc.ac.kr

Received 17 August 2021; Revised 26 October 2021; Accepted 29 October 2021; Published 22 November 2021

Academic Editor: Mateusz Maciejczyk

Copyright (c) 2021 Sungdam Han et al. This is an open access article distributed under the Creative Commons Attribution License, which permits unrestricted use, distribution, and reproduction in any medium, provided the original work is properly cited.

Chronic kidney disease-mineral bone disorder (CKD-MBD) is the most common complication in CKD patients. Although there is a consensus on treatment guidelines for CKD-MBD, it remains uncertain whether these treatment recommendations reflect actual practice. Therefore, the aim of this study was to investigate the CKD-MBD medication trend in real-world practice. This was a retrospective and observational study using a 12-year period database transformed into a common data model from three tertiary university hospitals. Study populations were subjects initially diagnosed as CKD. The date of diagnosis was designated as the index date. New patients were categorized year to year from 2008 to 2019 with a fixed observation period of 365 days to check the prescription of CKD-MBD medications including calcium-containing phosphate binder, noncalciumcontaining phosphate binder, aluminium hydroxide, vitamin D receptor activator (VDRA), and cinacalcet. The numbers of CKD patients in the three hospitals were 7555,2424 , and 5351, respectively. The proportion for patients with CKD-MBD medication prescription decreased yearly regardless of hospital and CKD stage ( $p$ for trend $<0.05)$. The use of aluminium hydroxide disappeared steadily while the use of VDRA increased annually in all settings. Despite these changes in prescription patterns, the mean value for CKD-MBD-related serologic markers was almost within target range. The proportion of the population within the target value was not significantly changed. Irrespective of hospital and CKD stage, similar trends of prescription for CKD-MBD medications were observed in real-world practice. Further research with a distributed network study may be helpful to understand medication trends in CKD-MBD treatment. 


\section{Introduction}

Chronic kidney disease-mineral bone disorder (CKD-MBD) is the most common complication in CKD patients. Vascular calcifications, biochemical abnormalities, and bone abnormalities constitute the CKD-MBD syndrome. Uncontrolled CKD-MBD causes serious changes, potentially disabling the body with complications including parathyroid hyperplasia, bone pain, fractures, vascular calcification, and even cardiovascular problems [1]. Furthermore, CKDMBD is ultimately associated with increased mortality [2]. Management of CKD-MBD has changed significantly over the past 20 years. In the 1990s, calcium-containing phosphate binders and calcitriol were increasingly used in the management of CKD-MBD. Due to concerns about hypercalcemia and oversuppression parathyroid hormone (PTH), a synthetic vitamin $\mathrm{D}$ analogue (paricalcitol), a noncalcium-containing phosphate binder (sevelamer), and a calcimimetic (cinacalcet) have entered the market since 1998, 1998, and 2004, respectively.

Kidney Disease Improving Global Outcomes (KDIGO) is a nonprofit foundation established in 2003 for patients with kidney diseases. It provides treatment strategies for patients with chronic kidney disease. KDIGO published the 2017 guidelines for CKD-MBD, an update of the guidelines first published in 2009 [3, 4]. A randomized controlled study in 2012 showed that when noncalcium-containing phosphate binders were administered to patients before dialysis, survival benefits were found compared to calciumcontaining phosphate binders [5]. In addition, in a study conducted on hemodialysis patients, the risk of death from arrhythmia and cardiovascular disease was significantly lower in patients taking noncalcium-containing phosphate binders than in patients using calcium-containing binders [6]. Based on these results, the $2017 \mathrm{KDIGO}$ guidelines recommended limiting the use of calcium-containing phosphate binders to control hyperphosphatemia in all adult patients at CKD stages 3 a to $5[3,7]$. The guidelines also recommended that CKD-MBD treatment strategies should be determined based on calcium, phosphate, and PTH concentrations in patients with CKD stages 3 a to 5 . They suggested that treatment should not be decided based on the results of a single test but should be determined based on results of regular measurements. As a result of analyzing a large cohort of dialysis patients, alcium, phosphate and PTH concentrations correlate and influence each other, so a treatment strategy tailored to each patient is needed [8]. The KDIGO guidelines are the most commonly used guidelines in the world, including in Korea.

Although there is a consensus on treatment guidelines, whether treatment recommendations reflect actual practice and whether there is any difference between actual practice and recommendations remain unclear. To identify these real-world prescription patterns, we used a common data model (CDM) that could allow for a systematic analysis of disparate observational databases [9]. Assessment of current prescription options for CKD-MBD using CDM in multiple hospitals will provide real evidence of common practice and treatment patterns. Therefore, the aim of this study was to show the applicability and feasibility of CDM in big data analysis for CKD-MBD. The primary goal of our study is to find out how well the current treatment pattern is consistent with the 2017 KDIGO guidelines. We also aimed to provide detailed information on the prescribing patterns of CKD-MBD and the differences between CKD stages and hospitals with changes in treatment trends from 2008 to 2019.

\section{Materials and Methods}

2.1. Data Source and Study Population. This was a retrospective and observational study using a 12 -year period database from three tertiary university hospitals. Data were transformed with Observational Medical Outcomes Partnership (OMOP) CDM, version 5.3, by the Observation Health Data Sciences and Informatics (OHDSI) network (https://www .ohdsi.org/) [10]. The concept of CDM was to transform data into common representation (terminologies, vocabularies, and coding schemes) and common format (data model) and to perform systematic analyses using a library of standard analytic tools $[9,11]$. It supports the access of multidatabase studies and translation of evidence across the individual hospitals and settings. The OMOP CDM applies standard vocabularies to normalize the meaning of data, such as Standard Nomenclature of Medicine (SNOMED) to represent clinical data, RxNorm to represent drugs, and Logical Observation Identifiers Names and Code (LOINC) to represent clinical measurements [12]. The CDM and open-source tools are opened on OHDSI GitHub (https:// github.com/OHDSI/), and discussions are opened on a dedicated open forum (https://forums.ohdsi.org/). More information about OHDSI and the analytical pipelines is described in The Book of OHDSI [13].

We performed two main analyses with different study populations. First, we analyzed the CKD-MBD prescription pattern of CKD patients from three distinct hospitals. The study populations included subjects initially diagnosed as CKD with any SNOMED clinical terms that matched to N18.0, N18.1, N18.2, N18.3, N18.4, N18.5, N18.9, N19, Z49, Z94.0, or Z99.2 of the ICD-10 code [14-16]. The date of diagnosis was designated as the index date. Subjects were limited to those with age greater than or equal to 20 years. To investigate the trend for prescription of CKD-MBD drugs, new patients were categorized year to year from 2008 to 2019 with a fixed observation period of 365 days. Second, to further investigate the prescription pattern according to the CKD stage, subjects with the estimated glomerular filtration rate (eGFR) in one hospital database were categorized. The eGFR was calculated using the modification of diet in the renal disease equation [17]. To avoid counting CKD patients repeatedly and to evaluate medication pattern for the first diagnosis of CKD, we only include patients having the first diagnosis of CKD with at least one measurement of eGFR between 30 days before and 30 days after the index date. In addition, end-stage kidney disease (ESKD) patients with dialysis were subclassified and further analyzed. Details about cohort definitions and concept set definitions including diseases, procedures (SNOMED), measurements 
(LOINC), and medications (RxNorm) are described in Supplementary Material Appendixes 1 and 2.

2.2. Characterization for Study Population. We used the ATLAS program, which is a free, public, and web-based software developed by the OHDSI community that helps the design and analysis with standard, patient-level, and observational CDM format data [18]. In the ATLAS program, database-level characterization can be automatically performed, including a description of age, medical history, and medication use. In addition to baseline characteristics, the Charlson comorbidity index (CCI) was calculated based on the underlying disease, including myocardial infarction, congestive heart failure, peripheral vascular disease, cerebrovascular disease, dementia, chronic pulmonary disease, connective tissue disease, peptic ulcer, mild liver disease, diabetes with and without complications, paraplegia or hemiplegia, renal disease, any or metastatic cancer, moderate or severe liver disease, and acquired immune deficiency syndrome before the start of the follow-up period [19].

2.3. CKD-MBD-Related Medications. CKD-MBD-related medications were categorized as calcium-containing phosphate binder (calcium carbonate and calcium acetate), noncalcium-containing phosphate binder (sevelamer and lanthanum), aluminium hydroxide, vitamin $\mathrm{D}$ receptor activator (VDRA), and cinacalcet. The percentage of prescriptions for CKD-MBD-related medications was subsequently estimated yearly.

2.4. Open-Source Treatment Pathway. In this study, the treatment pathway in OHDSI [20], an open-source software with ATLAS, was used. For each patient in the cohort, this program identifies the sequence of medications in the RxNorm ingredient level, arranging them by first exposure to medications. If one CKD patient has at least one CKDMBD-related medication, this patient is included in the group of persons with the treatment pathway. Conversely, the other CKD patient has no CKD-MBD-related medications; this patient is included in the group of persons without the treatment pathway. After each patient's sequence of medications was built, the number of patients with the same treatment sequence was counted and expressed by sunburst plots. These sequences were limited to 10 medications.

2.5. Calcium, Phosphate, and Parathyroid Hormone. Serum concentrations of calcium, phosphate, and albumin were determined with automated and standardized methods. Intact PTH (iPTH) concentration was measured with second-generation PTH assays at the hospital. Final laboratory values of calcium, phosphate, albumin, and iPTH within the follow-up period for CKD stage 5 patients were extracted. Corrected calcium was calculated with albumin as Equation (1) when the albumin level was less than $4.0 \mathrm{~g} /$ $\mathrm{dL}$. Target ranges for this study were as follows: calcium, 8.6 to $10.2 \mathrm{mg} / \mathrm{dL}$; serum phosphate, 2.5 to $4.5 \mathrm{mg} / \mathrm{dL}$; and $\mathrm{iPTH}, 130$ to $600 \mathrm{pg} / \mathrm{mL}$. These were recommended by the 2017 CKD-MBD KDIGO guidelines and a multicentre study in Korea [3, 21, 22].
Corrected calcium $=$ Total calcium $+0.8 \times(4.0-$ Albumin $)$.

2.6. Analytical and Statistical Methods. This was a descriptive study for CKD-MBD medications according to the hospital and CKD stage. Continuous variables are presented as mean and standard deviation. Categorical variables are expressed as number and percentage. To refine and analyze data, Structured Query Language and R programming language version 4.1.0 ( $\mathrm{R}$ foundation for Statistical Computing, Vienna, Austria) were implemented. To compare the characteristics of CKD patients for the three different hospitals, the one-way analysis of variance and the chi-square test were performed. To evaluate the prescription trend in the different hospitals and CKD stages, the Cochran-Armitage trend test was performed. Statistical analyses were performed with $\mathrm{R}$ version 4.1.0. The $p$ value $<0.05$ was considered statistically significant.

2.7. Ethical Consideration. Since all databases transformed into CDM were deidentified, this study was approved by the Institutional Review Board of Ajou University Hospital (approval no. AJIRB-MED-MDB-21-241) with a waiver for informed consent.

\section{Results}

3.1. Baseline Characteristics of Subjects. Table 1 shows the baseline characteristics of subjects from three different hospitals. The numbers of CKD patients in the three hospitals were 7555,2424 , and 5351, respectively. The proportion of those over 80 years of age was higher in the B and C hospitals (19.83\% and $20.73 \%$, respectively) than in the A hospital $(13.42 \%)$ with a significant $p$ value $(<0.001)$. Males accounted for $58 \%$ to $60 \%$. Subjects from the A hospital had more diabetes and CKD stage 5. Subjects from the C hospital had more dyslipidemia and cerebrovascular disease with a higher Charlson comorbidity index (all $p$ values < 0.001). Regarding medication use, subjects from the B hospital had more medication prescriptions except for opioids and corticosteroids. Overall, there were significant differences in baseline characteristics from three different hospitals except for sex.

3.2. Yearly CKD-MBD Medication Prescription Pattern in CKD Patients from Three Different Hospitals. Yearly prescription patterns in CKD patients from 3 different hospitals are shown in Figure 1. Although proportions of CKD-MBD medication prescriptions were different among the three hospitals, these proportions decreased yearly (all $p$ for trend $<0.05)$. Proportions of CKD-MBD medication prescriptions in 2019 were roughly half of those in 2008 in all hospitals. Regarding classes of CKD-MBD medications, the percentage of calcium-containing phosphate binder decreased dramatically from 2008 to 2019 (all $p$ for tend $<0.05$ ), while the percentage for noncalcium-containing phosphate binder increased gradually from 2018. The use of aluminum hydroxide disappeared steadily while the use of VDRA 
TABLE 1: Baseline characteristics of subjects from three different hospitals.

\begin{tabular}{|c|c|c|c|c|}
\hline Baseline characteristics & A hospital $(n=7555)$ & B hospital $(n=2424)$ & C hospital $(n=5351)$ & $p$ value \\
\hline \multicolumn{5}{|l|}{ Age group (\%) } \\
\hline $20-24$ & $<1$ & $<1$ & $<1$ & $\mathrm{NA}^{\dagger}$ \\
\hline $25-29$ & $<1$ & $<1$ & $<1$ & \\
\hline $30-34$ & 1.51 & $<1$ & 1.18 & \\
\hline $35-39$ & 2.33 & 2.06 & 2.11 & \\
\hline $40-44$ & 3.87 & 2.35 & 2.63 & \\
\hline $45-49$ & 6.17 & 4.00 & 4.18 & \\
\hline $50-54$ & 8.86 & 6.60 & 5.49 & \\
\hline $55-59$ & 9.48 & 9.60 & 8.83 & \\
\hline $60-64$ & 10.84 & 10.14 & 10.20 & \\
\hline $65-69$ & 12.10 & 13.03 & 11.75 & \\
\hline $70-74$ & 15.02 & 15.25 & 14.51 & \\
\hline $75-79$ & 14.03 & 15.05 & 16.19 & \\
\hline $80-84$ & 9.73 & 11.01 & 13.05 & \\
\hline Over 85 & 3.69 & 8.82 & 7.68 & \\
\hline Sex $(\%)$ & & & & 0.14 \\
\hline Male & 60.54 & 59.93 & 58.79 & \\
\hline Female & 39.46 & 40.07 & 41.21 & \\
\hline \multicolumn{5}{|l|}{ Medical history (\%) } \\
\hline Hypertension & 52.68 & 41.47 & 60.3 & $<0.001$ \\
\hline Diabetes & 31.06 & 17.89 & 26.85 & $<0.001$ \\
\hline Dyslipidemia & 6.32 & 5.56 & 20.5 & $<0.001$ \\
\hline Cerebrovascular disease & 9.17 & 8.45 & 14.98 & $<0.001$ \\
\hline Heart failure & 5.12 & 7.46 & 5.08 & $<0.001$ \\
\hline CKD stage $5^{\ddagger}$ & 14.29 & 6.64 & 8.66 & $<0.001$ \\
\hline CKD stage $4^{\ddagger}$ & 8.52 & 7.75 & 12.94 & $<0.001$ \\
\hline CKD stage $3^{\ddagger}$ & 3.29 & 24.32 & 30.77 & $<0.001$ \\
\hline Charlson comorbidity index, mean (SD) & $4.06(2.30)$ & $4.15(1.94)$ & $4.40(2.27)$ & $<0.001$ \\
\hline \multicolumn{5}{|l|}{ Medication use (\%) } \\
\hline Agents acting on the renin-angiotensin system & 50.06 & 61.17 & 58.21 & $<0.001$ \\
\hline Beta-blocking agents & 36.70 & 48.43 & 41.98 & $<0.001$ \\
\hline Calcium channel blockers & 50.42 & 61.29 & 56.53 & $<0.001$ \\
\hline Diuretics & 47.20 & 63.36 & 59.2 & $<0.001$ \\
\hline Antidiabetic drugs & 39.96 & 53.71 & 47.58 & $<0.001$ \\
\hline Lipid-modifying agents & 59.26 & 71.39 & 61.61 & $<0.001$ \\
\hline Antithrombotic agents & 52.24 & 67.31 & 61.62 & $<0.001$ \\
\hline Opioids & 57.67 & 62.94 & 63.96 & $<0.001$ \\
\hline Anti-inflammatory and antirheumatic products & 65.25 & 76.59 & 76.06 & $<0.001$ \\
\hline Corticosteroids & 37.81 & 39.86 & 43.47 & $<0.001$ \\
\hline
\end{tabular}

${ }^{\dagger}$ To protect individual data, results under the $1 \%$ cannot be calculated in the ATLAS program. Chi-square test for the age group cannot be performed. ${ }^{*}$ In a table, CKD stage was categorized by only Standard Nomenclature of Medicine (SNOMED) diagnosis code (CKD stage 5: 443611; CKD stage 4: 443612; CKD stage 3: 443597). Abbreviations: CKD: chronic kidney disease; NA: not applicable; SD: standard deviation.

increased annually (all $p$ for trend $<0.05$ ). These patterns of prescription were found for all hospitals.

3.3. Yearly CKD-MBD Medication Prescription Pattern for CKD Patients according to CKD Stage. Yearly prescription patterns for CKD patients according to the CKD stage with the diagnosis code and eGFR in the A hospital are shown in Figure 2. The numbers of CKD patients according to the CKD stage were 4154 for CKD stage 3, 1622 for CKD stage 4, and 1779 for CKD stage 5. Proportions of CKDMBD medication prescriptions decreased yearly regardless of CKD stage (all $p$ for trend <0.05). In particular, reduced fractions of prescriptions from 2008 to 2019 were as follows: $28 \%$ for CKD stage $3,17 \%$ for CKD stage 4 , and $14 \%$ for 

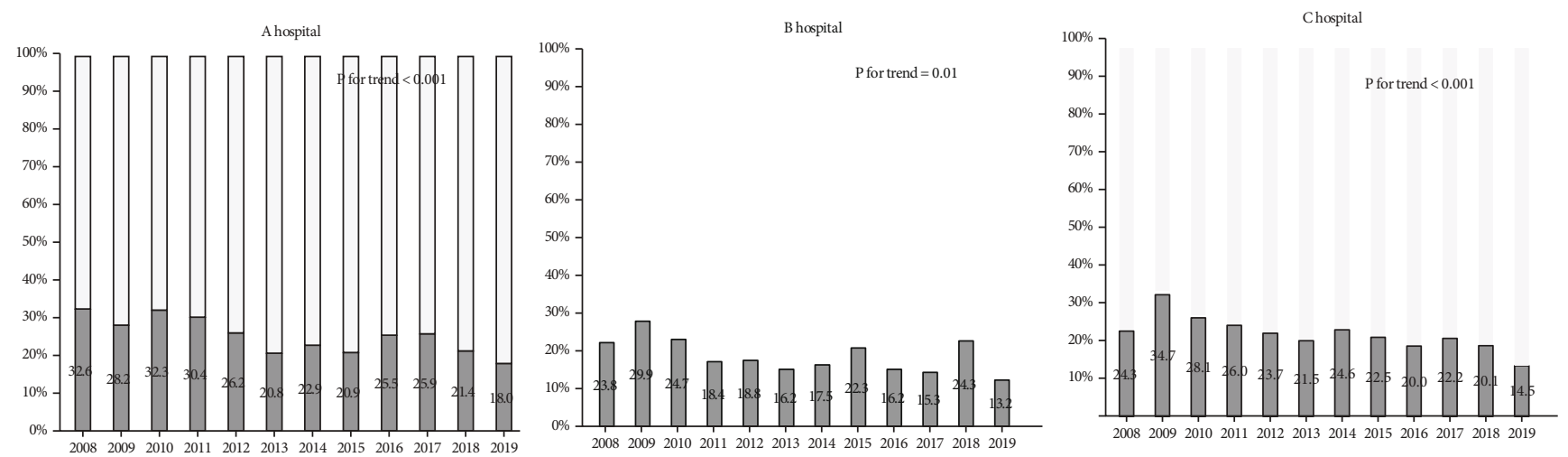

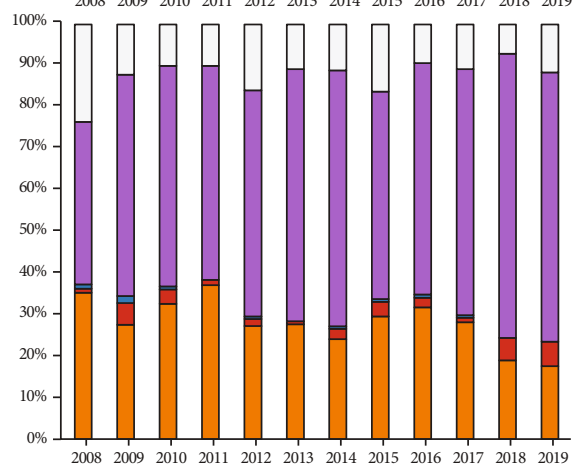

(a)

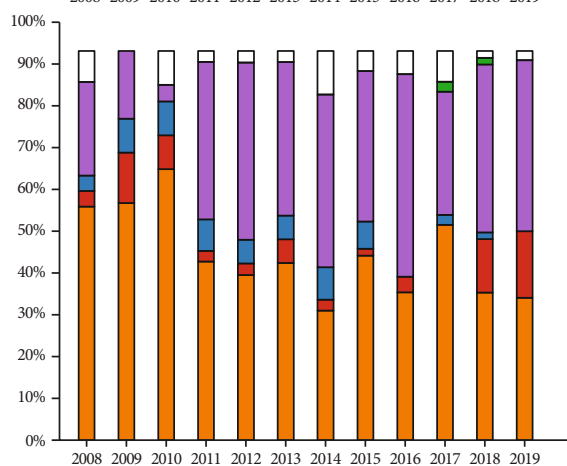

(b)

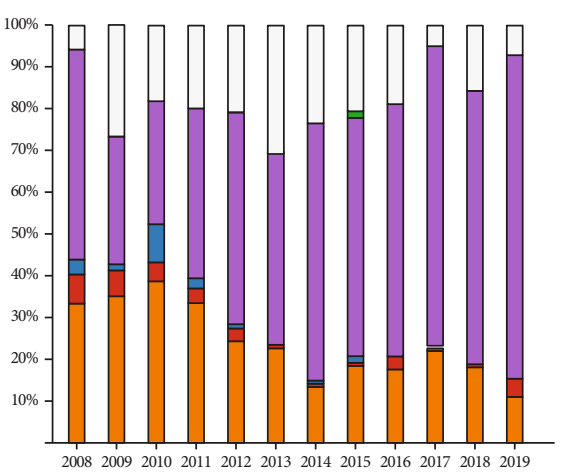

(c)

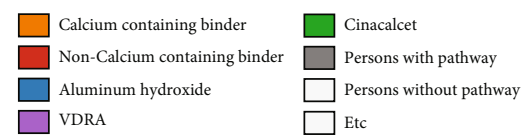

FIGURE 1: Yearly CKD-MBD medication prescription patterns for CKD patients from three different hospitals. Abbreviations: CKD-MBD: chronic kidney disease-mineral bone disorder; VDRA: vitamin D receptor activator.

CKD stage 5. For CKD stage 3, the percentage of calciumcontaining phosphate binder was $1.4 \%$ in 2019 . For CKD stages 4 and 5 , percentages of calcium-containing phosphate binder tended to decrease over time but were statically nonsignificant ( $p$ for trend $=0.50$ and 0.08 , respectively). The use of aluminum hydroxide disappeared while the use of VDRA increased over time for those with CKD stage 5 (all $p$ for trend $<0.05)$. The proportion for CKD-MBD medication in ESKD patients with dialysis also decreased from 2008 to 2019. The prescription pattern for ESKD patients with dialysis is described in Supplementary Figure S1.

3.4. Treatment Pathway for CKD-MBD Medication according to CKD Stage. The treatment pathway for CKDMBD medication according to the CKD stage is shown in Figure 3. Regarding the first prescription of CKD-MBD medication, the percentage of calcium-containing phosphate binder decreased while the percentage of noncalciumcontaining phosphate binder increased from 2008 to 2019 (all $p$ for trend $<0.05$ ). In particular, the prescription of the noncalcium-containing phosphate binder appeared as a secondary or tertiary prescription in 2008 but appeared increasingly as the first prescription in 2019. The use of aluminum hydroxide disappeared while the use of VDRA increased from 2008 to 2019 (all $p$ for trend < 0.05). The treatment pathway for CKD-MBD medication in ESKD patients with dialysis is shown in Supplementary Figure S1.
3.5. CKD-MBD-Related Serologic Markers of CKD Stage 5 Patients in Hospital A. To evaluate the effect of mutational trends of CKD-MBD medication, values of calcium, phosphate, calcium $\times$ phosphate, and $\mathrm{iPTH}$ in $\mathrm{CKD}$ stage 5 patients were investigated in hospital A. CKD-MBDrelated serologic markers are shown in Figure 4. Mean values of calcium $\times$ phosphate product were 32.3 to $34.8 \mathrm{mg} / \mathrm{dL}$ (standard deviation (SD) 5.7 to 7.0). Mean values of iPTH were 119.1 to $198.3 \mathrm{pg} / \mathrm{mL}$ (SD 72.5 to 295.2). The proportion of the population within the target value ranged from $44.0 \%$ to $48.1 \%$, showing no significant change from 2008 to 2019 ( $p$ for trend $>0.05$ ).

\section{Discussion}

This study evaluated the pattern of CKD-MBD medication using the CDM database from three different hospitals. Approximately, the proportion of patients with CKD-MBD medication prescription decreased yearly regardless of hospital and CKD stage. The use of aluminum hydroxide disappeared steadily while the use of VDRA increased annually in all settings. Despite these changes in prescription patterns, mean values of CKD-MBD-related serologic markers were within their target ranges and the proportion of the population within these target values was not significantly changed.

Patients from these three hospitals showed different baseline characteristics. There were differences in the 


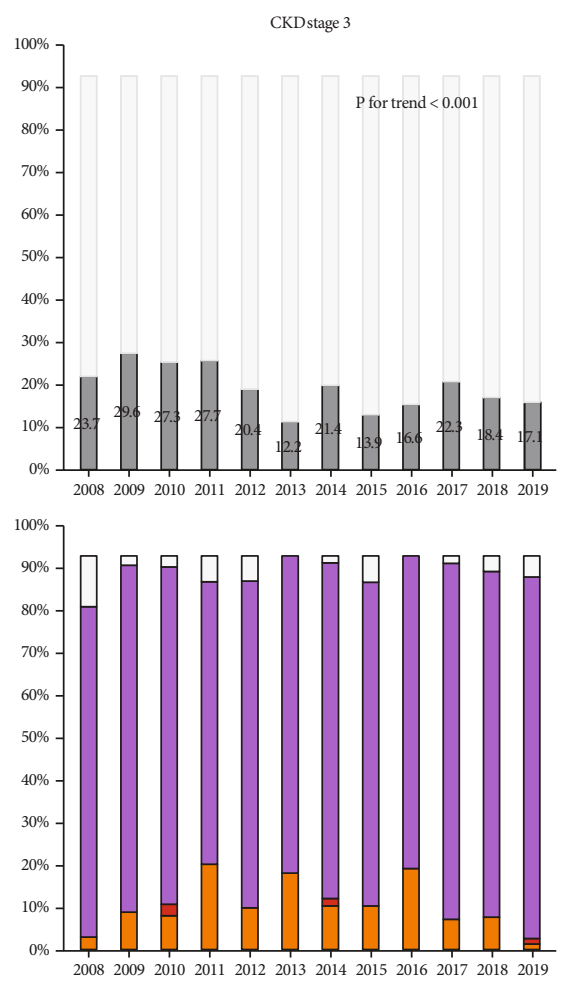

(a)

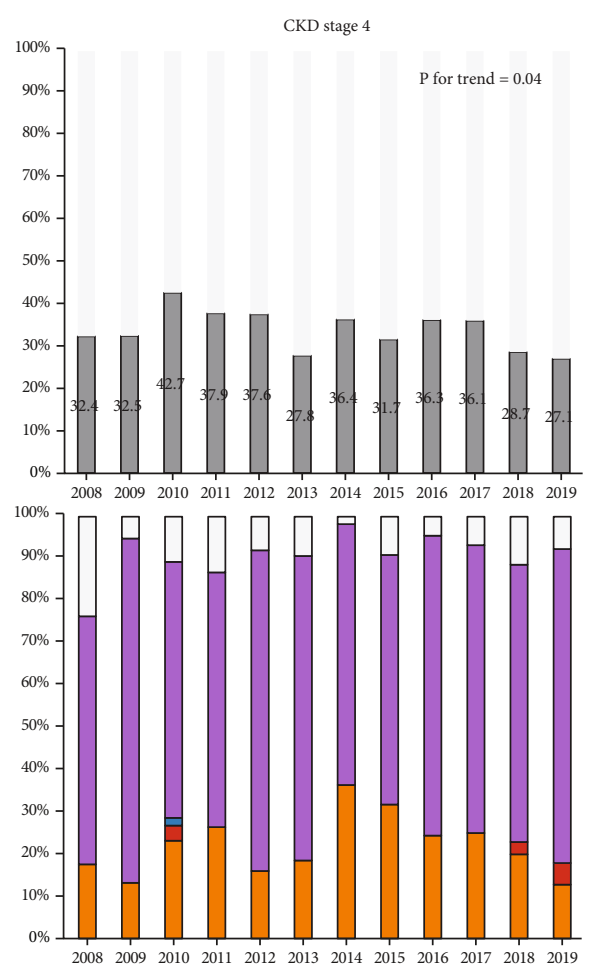

(b)

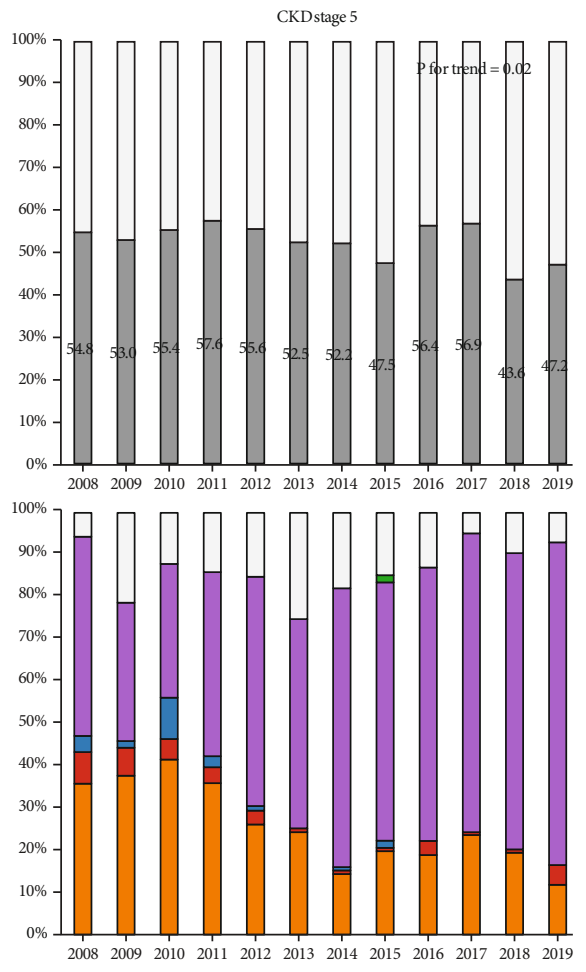

(c)

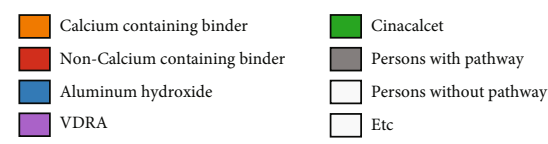

FIGURE 2: Yearly CKD-MBD medication prescription patterns for CKD patients according to CKD stage. Abbreviations: CKD-MBD: chronic kidney disease-mineral bone disorder; VDRA: vitamin D receptor activator.

proportion of patient age, past medical history, CKD stage, and medication history. According to the Korean Renal Data System registry results for 2019 [23], diabetes accounts for about $50 \%$ of ESKD patients, and these fractions are very different from the above baseline characteristics. Such differences among these three hospitals have an influence on the community where these hospitals are located. In addition, it seemed that there was a difference in the behavior of entering diagnosis codes for each hospital.

According to the results of our study on drug prescription proportion, there were differences between hospitals. However, the overall prescription proportion decreased. Several reasons can be considered for such decrease. First, this decrease could be attributable to an increase in overall CKD prevalence [24]. According to data released by the Korean National Health Insurance Service in 2015, the prevalence of CKD patients increased by $13.6 \%$ between 2009 and 2013. Causes include an increase in the elderly population and an increase in the number of chronic diseases such as hypertension and diabetes. Another cause might be early detection of CKD patients through screening. About $15 \%$ of newly increased patients with CKD were treated at tertiary university hospitals [25]. Among them, the patients who did not need CKD-MBD treatment were included, which might have led to our study results. Second, the prescription was reduced by establishing nutrition education such as a phosphate restriction diet. Several studies have reported that a phosphate restriction diet is helpful for hyperphosphatemia [26]. Despite some limitations, the KDIGO 2017 phosphate restriction diet is useful for treating hyperphosphatemia. Third, in 2009 and 2017, the KDIGO CKD-MBD guideline was published $[3,4]$. It was thought that the use of unnecessary prescription for CKD-MBD was reduced through evidence-based medicine.

In South Korea, CKD is regarded as one of the most significant health problems with the highest cost per patient [27]. All South Korean citizens were covered by the national health insurance (NHI), which reviews most of medical care including medication. For CKD-BMD prescription, although the $\mathrm{NIH}$ covers the use of noncalcium-containing phosphate binder to only ESKD patients as shown in the results of this study, the proportion of those using noncalcium-containing phosphate binder is gradually increasing at all CKD stages. It appeared to be increasingly used as the first medication. This seems to be the result of physicians choosing medications according to the 2017 KDIGO CKD-MBD guidelines even if insurance issues are taken into account. In 2018, the NIH relaxed the insurance coverage for noncalcium-containing phosphate binder. However, it still limited the treatment target to ESKD patients.

As shown in this study, the use of aluminum hydroxide for limiting phosphate absorption was deprecated. Long- 
Treatment pathway for CKD-MED medication

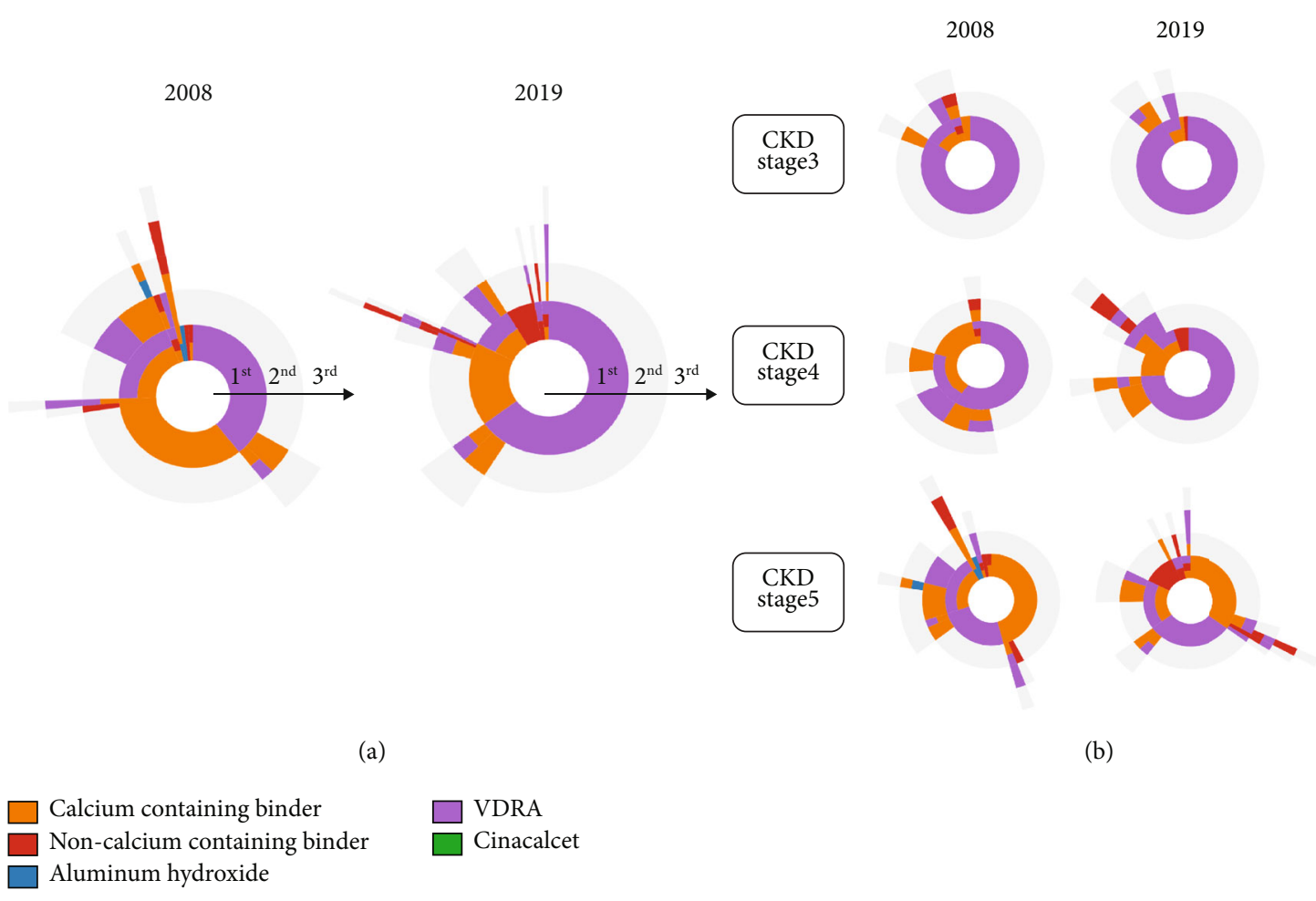

FIgure 3: Treatment pathway for CKD-MBD medication according to CKD stage. Abbreviations: CKD-MBD: chronic kidney diseasemineral bone disorder; VDRA: vitamin $\mathrm{D}$ receptor activator.

term use of aluminum hydroxide is known to be associated with side effects such as osteomalacia and encephalopathy caused by aluminum. Its use seems to have gradually decreased accordingly [28]. PTH elevation may represent a compensatory response to hyperphosphatemia and increasing bone resistance to PTH. Moreover, in two past randomized trials of VDRA in CKD patients, the use of VDRA showed a decrease in PTH but no change in cardiovascular end points with an increased risk of hypercalcemia [29, 30]. For these reasons, the 2017 KDIGO CKD-MBD guidelines recommend that VDRA should not be routinely used for those with abnormal PTH levels. However, in our study, VDRA use showed a gradual increase. In a previous study, since vitamin $\mathrm{D}$ deficiency is common in CKD patients $[31,32]$, it is thought that the prescription of nutritional vitamin D (cholecalciferol and ergocalciferol) has increased. In this study, since VDRA was investigated as both active vitamin $\mathrm{D}$ and nutritional vitamin $\mathrm{D}$, further studies are needed in the future. In spite of changing treatment trends according to guidelines, in our study, the proportion of patients included in the treatment target did not differ significantly from 2008 to 2019. Of course, since the use of noncalcium-containing phosphate binder is associated with survival benefits and reduced risk of death from arrhythmia and cardiovascular disease $[5,6]$, laboratory tests alone cannot determine the usefulness guideline. However, several studies have recently shown no significant difference in composite cardiovascular events between the use of calcium-containing phosphate binder and noncalcium- containing phosphate binder $[33,34]$. In terms of medical costs, a noncalcium-containing phosphate binder costs nearly 20-30 times more than a calcium-containing phosphate binder, which is a significant burden on both the $\mathrm{NIH}$ and individual patients. Given the growing trend in the use of noncalcium-containing phosphate binder, its benefits need to be further elucidated.

In this study, CDM-based analysis suitable for big data analysis was used. With CDM, we could perform analyses in the distributed region and include a large number of CKD patients. If the traditional method of reviewing medical records was used, it would be very laborious and subject to human errors. This study confirmed the applicability and feasibility of CDM-based analysis and verified the possibility of using it for a future distributed network study in the field of nephrology. However, this study has some limitations. First, because a deidentified database was used in this study, we could not review the medical records of individual patients or consider their specific situations. Second, although the exact diagnosis for CKD was necessary, we used diagnostic codes based on the definition of CKD. This definition issue is a common problem in CDM-based analysis. Therefore, we used the CKD definition, which was used in previous studies [14-16]. Third, to investigate the pattern of prescription according to CKD stage, subclassification of CKD stages using eGFR values was performed, but subgrouping was performed only in the A hospital. In addition, individual for CKD stages can be varied during the followup period. However, according to a 10 -year observational 


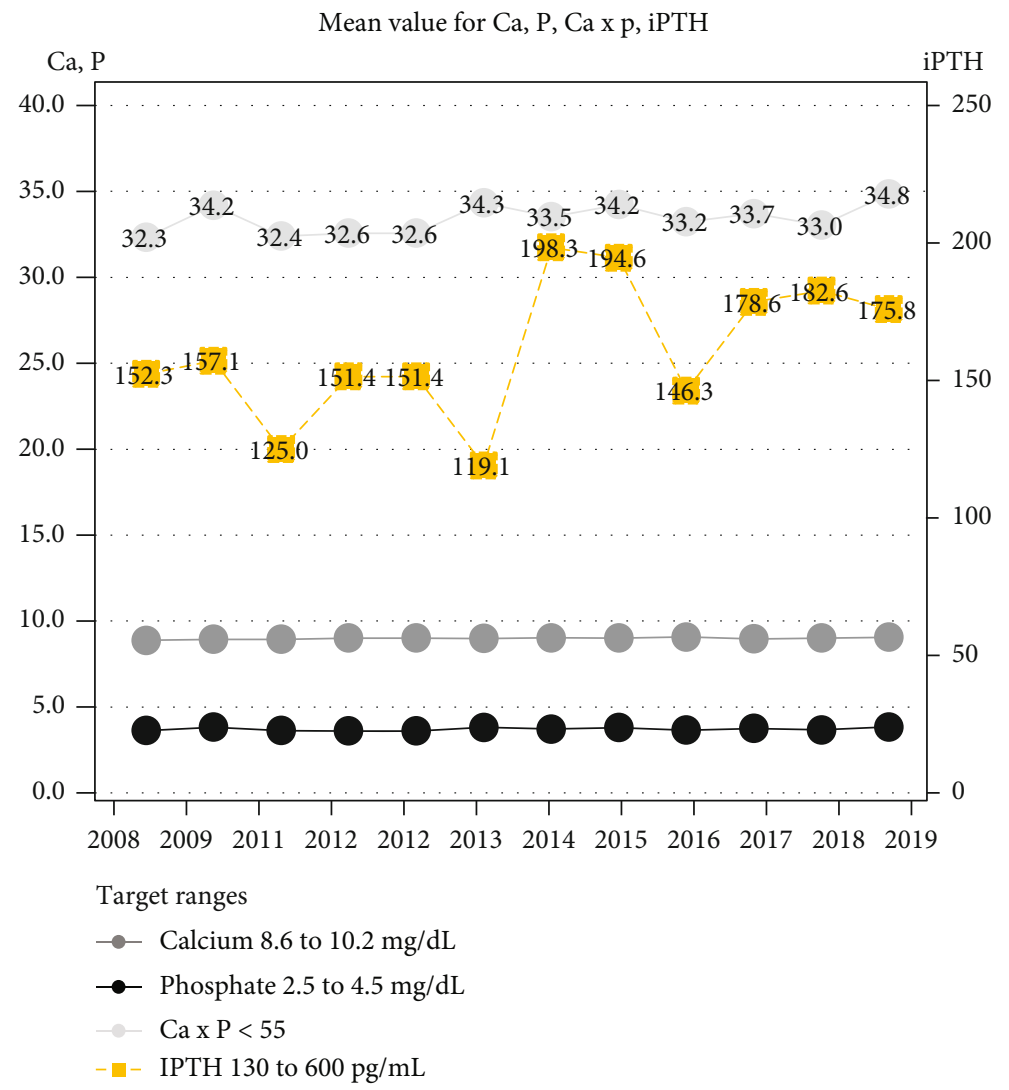

(a)

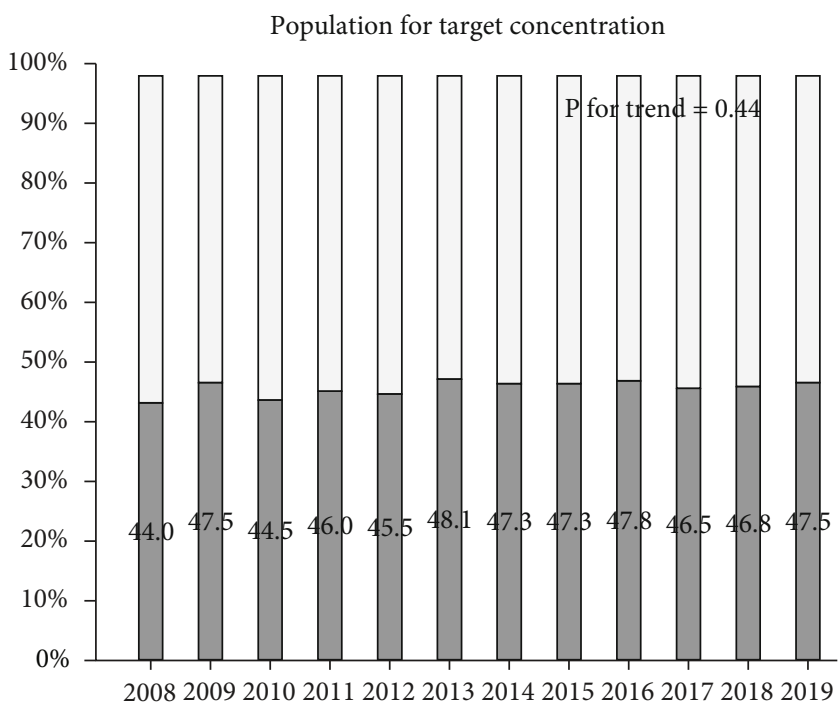

(b)

FIGURE 4: CKD-MBD-related serologic markers in patients with CKD at stage 5. Target levels: calcium, 8.4 to $9.6 \mathrm{mg} / \mathrm{dL}$; phosphate, 2.4 to $5.0 \mathrm{mg} / \mathrm{dL}$; Ca $\times \mathrm{P}$ product, $<55 \mathrm{mg}^{2} / \mathrm{dL}^{2}$; and $\mathrm{iPTH}, 100$ to $300 \mathrm{pg} / \mathrm{mL}$. Abbreviations: CKD-MBD: chronic kidney disease-mineral bone disorder; iPTH: intact parathyroid hormone.

study of Asian CKD patients in the past, the rate of eGFR decline in the participants was $0.36 \mathrm{~mL} / \mathrm{min} / 1.73 \mathrm{~m}^{2} /$ year on average [35]. The number of patients whose CKD stage has changed over the course of one year is thought to be small. Fourth, the association between reduced use of calcium-containing phosphate binder and cardiovascular outcomes was not investigated. Finally, we enrolled patients from three different hospitals, but we cannot extract the laboratory data in all hospitals. This is the common problem using CDM due to the limitation of the current version of OHDSI CDM. Considering these limitations, further external validation is needed to confirm our results. 


\section{Conclusions}

In summary, this is the first study to investigate prescription patterns of CKD-MBD in Korea using CDM. The proportion of patients with CKD-MBD medication prescription decreased yearly regardless of hospital and CKD stage. However, mean values for CKD-MBD-related serologic markers were not significantly changed for the study period. Based on results of this study, further research with a distributed network study may be helpful to understand medication trends in CKD-MBD treatment.

\section{Data Availability}

The data used during the present study are available from the corresponding author upon reasonable request.

\section{Disclosure}

The study sponsor had no role in the study design, data analysis, or data interpretation.

\section{Conflicts of Interest}

The authors declare that there is no conflict of interest regarding the publication of this paper.

\section{Authors' Contributions}

Sungdam Han and Minkook Son contributed equally as cofirst authors.

\section{Acknowledgments}

We are thankful to FeederNet for providing the Health Big Data Platform. This work was supported by a grant (No. 2019R1G1A1100671) of the National Research Foundation of Korea (NRF) funded by the Korea government (MSIT). This research was also supported by the Bio Industrial Strategic Technology Development Program (20003883, 20005021) funded by the Ministry of Trade, Industry and Energy (MOTIE, Korea) and a grant (HR16C0001) from the Korea Health Technology R\&D Project through the Korea Health Industry Development Institute (KHIDI) funded by the Ministry of Health and Welfare, Republic of Korea.

\section{Supplementary Materials}

Appendix 1: cohort definition. Appendix 2: concept set definitions. Figure S1: yearly CKD-MBD medication prescription pattern and treatment pathway for ESKD patients. (Supplementary Materials)

\section{References}

[1] J. J. Kazama, "Chronic kidney disease and fragility fracture," Clinical and Experimental Nephrology, vol. 21, Suppl 1, pp. 46-52, 2017.
[2] T. Isakova, "Comparison of mineral metabolites as risk factors for adverse clinical outcomes in CKD," Seminars in Nephrology, vol. 33, no. 2, pp. 106-117, 2013.

[3] M. Ketteler, G. A. Block, P. Evenepoel et al., "KDIGO 2017 Clinical Practice Guideline Update for the Diagnosis, Evaluation, Prevention, and Treatment of Chronic Kidney DiseaseMineral and Bone Disorder (CKD-MBD)," Kidney International Supplements, vol. 7, no. 1, pp. 1-59, 2017.

[4] Kidney Disease: Improving Global Outcomes (KDIGO) CKDMBD Work Group, "KDIGO clinical practice guideline for the diagnosis, evaluation, prevention, and treatment of chronic kidney disease-mineral and bone disorder (CKD-MBD)," Kidney International Supplement, vol. 113, pp. S1-S130, 2009.

[5] B. di Iorio, A. Bellasi, D. Russo, and on behalf of the INDEPENDENT Study Investigators, "Mortality in kidney disease patients treated with phosphate binders: a randomized study," Clinical Journal of the American Society of Nephrology, vol. 7, no. 3, pp. 487-493, 2012.

[6] B. di Iorio, D. Molony, C. Bell et al., "Sevelamer versus calcium carbonate in incident hemodialysis patients: results of an open-label 24-month randomized clinical trial," American Journal of Kidney Diseases, vol. 62, no. 4, pp. 771-778, 2013.

[7] T. Isakova, T. L. Nickolas, M. Denburg et al., "KDOQI US commentary on the 2017 KDIGO clinical practice guideline update for the diagnosis, evaluation, prevention, and treatment of chronic kidney disease-mineral and bone disorder (CKD-MBD)," American Journal of Kidney Diseases, vol. 70, no. 6, pp. 737-751, 2017.

[8] G. A. Block, R. D. Kilpatrick, K. A. Lowe, W. Wang, and M. D. Danese, "CKD-mineral and bone disorder and risk of death and cardiovascular hospitalization in patients on hemodialysis," Clinical Journal of the American Society of Nephrology, vol. 8, no. 12, pp. 2132-2140, 2013.

[9] R. Makadia and P. B. Ryan, "Transforming the premier perspective hospital database into the Observational Medical Outcomes Partnership (OMOP) common data model," EGEMS (Washington, DC), vol. 2, no. 1, p. 15, 2017.

[10] G. Hripcsak, J. D. Duke, N. H. Shah et al., "Observational Health Data Sciences and Informatics (OHDSI): opportunities for observational researchers," Studies in Health Technology and Informatics, vol. 216, pp. 574-578, 2015.

[11] E. A. Voss, R. Makadia, A. Matcho et al., "Feasibility and utility of applications of the common data model to multiple, disparate observational health databases," Journal of the American Medical Informatics Association, vol. 22, no. 3, pp. 553-564, 2015.

[12] S. Kent, E. Burn, D. Dawoud et al., "Common problems, common data model solutions: evidence generation for health technology assessment," PharmacoEconomics, vol. 39, no. 3, pp. 275-285, 2021.

[13] H. Abedtash, M. Ascha, M. Beno et al., The Book of SHDSI: observational health data sciences and informatics, OHDSI community, 2021, https://ohdsi.github.io/TheBookOfOhdsi/.

[14] K. M. Kim, H. J. Oh, H. Y. Choi, H. Lee, and D. R. Ryu, "Impact of chronic kidney disease on mortality: a nationwide cohort study," Kidney Research and Clinical Practice, vol. 38, no. 3, pp. 382-390, 2019.

[15] M. K. Kim, K. Han, H. S. Kim et al., "Effects of variability in blood pressure, glucose, and cholesterol concentrations, and body mass index on end-stage renal disease in the general population of Korea," Journal of Clinical Medicine, vol. 8, no. 5, p. 755, 2019. 
[16] Y. H. Kim, J. G. Kang, S. J. Lee et al., "Underweight increases the risk of end-stage renal diseases for type 2 diabetes in Korean population: data from the National Health Insurance Service health checkups 2009-2017," Diabetes Care, vol. 43, no. 5, pp. 1118-1125, 2020.

[17] A. S. Levey, J. P. Bosch, J. B. Lewis, T. Greene, N. Rogers, and D. Roth, "A more accurate method to estimate glomerular filtration rate from serum creatinine: a new prediction equation. Modification of Diet in Renal Disease Study group," Annals of Internal Medicine, vol. 130, no. 6, pp. 461-470, 1999.

[18] "Chapter 8 OHDSI Analytics Tools: 8.3 ATLAS," in The Book of OHDSI: observational health data sciences and informaticsOHDSI communityOctober 2021, https://ohdsi.github.io/ TheBookOfOhdsi/OhdsiAnalyticsTools.html\#atlas.

[19] M. E. Charlson, P. Pompei, K. L. Ales, and C. R. MacKenzie, "A new method of classifying prognostic comorbidity in longitudinal studies: development and validation," Journal of Chronic Diseases, vol. 40, no. 5, pp. 373-383, 1987.

[20] G. Hripcsak, P. B. Ryan, J. D. Duke et al., "Characterizing treatment pathways at scale using the OHDSI network," Proceedings of the National Academy of Sciences of the United States of America, vol. 113, no. 27, pp. 7329-7336, 2016.

[21] G. H. Kim, B. S. Choi, D. R. Cha et al., "Serum calcium and phosphorus levels in patients undergoing maintenance hemodialysis: a multicentre study in Korea," Kidney Research and Clinical Practice, vol. 33, no. 1, pp. 52-57, 2014.

[22] K. Jin, T. H. Ban, J. Y. Jung et al., "Stabilization of serum alkaline phosphatase in hemodialysis patients by implementation of local chronic kidney disease-mineral bone disorder management strategy: a quality improvement study," Kidney Research and Clinical Practice, vol. 37, no. 2, pp. 157-166, 2018.

[23] Y. A. Hong, T. H. Ban, C. Y. Kang et al., "Trends in epidemiologic characteristics of end-stage renal disease from 2019 Korean Renal Data System (KORDS)," Kidney Research and Clinical Practice, vol. 40, no. 1, pp. 52-61, 2021.

[24] Global Burden of Disease Study 2013 Collaborators, "Global, regional, and national incidence, prevalence, and years lived with disability for 301 acute and chronic diseases and injuries in 188 countries, 1990-2013: a systematic analysis for the Global Burden of Disease Study 2013," Lancet, vol. 386, no. 9995, pp. 743-800, 2015.

[25] “2014 National Health Insurance Yearbook," Health insurance review \& assessment serviceAugust 2021, https://www.hira.or . $\mathrm{k} \mathrm{r} / \mathrm{b}$ b s D u m m y. d o ? p g m i d = HIRAA $20045020000 \&$ brdScnBltNo $=4 \&$ brdBltNo= 2307\&pageIndex=1\#none.

[26] C. Sullivan, S. S. Sayre, J. B. Leon et al., "Effect of food additives on hyperphosphatemia among patients with end-stage renal disease: a randomized controlled trial," JAMA, vol. 301, no. 6, pp. 629-635, 2009.

[27] S. H. Kim, M. W. Jo, D. S. Go, D. R. Ryu, and J. Park, "Economic burden of chronic kidney disease in Korea using national sample cohort," Journal of Nephrology, vol. 30, no. 6, pp. 787-793, 2017.

[28] A. C. Alfrey, "Aluminum toxicity in patients with chronic renal failure," Therapeutic Drug Monitoring, vol. 15, no. 6, pp. 593-597, 1993.

[29] A. Y. Wang, F. Fang, J. Chan et al., "Effect of paricalcitol on left ventricular mass and function in CKD-the OPERA trial," Journal of the American Society of Nephrology, vol. 25, no. 1, pp. 175-186, 2014.
[30] R. Thadhani, E. Appelbaum, Y. Pritchett et al., "Vitamin D therapy and cardiac structure and function in patients with chronic kidney disease: the PRIMO randomized controlled trial," JAMA, vol. 307, no. 7, pp. 674-684, 2012.

[31] A. Levin, G. L. Bakris, M. Molitch et al., "Prevalence of abnormal serum vitamin D, PTH, calcium, and phosphorus in patients with chronic kidney disease: results of the study to evaluate early kidney disease," Kidney International, vol. 71, no. 1, pp. 31-38, 2007.

[32] R. E. LaClair, R. N. Hellman, S. L. Karp et al., "Prevalence of calcidiol deficiency in CKD: a cross-sectional study across latitudes in the United States," American Journal of Kidney Diseases, vol. 45, no. 6, pp. 1026-1033, 2005.

[33] H. Ogata, M. Fukagawa, H. Hirakata et al., "Effect of treating hyperphosphatemia with lanthanum carbonate vs calcium carbonate on cardiovascular events in patients with chronic kidney disease undergoing hemodialysis: the LANDMARK randomized clinical trial," JAMA, vol. 325, no. 19, pp. 19461954, 2021.

[34] S. Habbous, S. Przech, R. Acedillo, S. Sarma, A. X. Garg, and J. Martin, "The efficacy and safety of sevelamer and lanthanum versus calcium-containing and iron-based binders in treating hyperphosphatemia in patients with chronic kidney disease: a systematic review and meta-analysis," Nephrology, Dialysis, Transplantation, vol. 32, no. 1, pp. 111-125, 2016.

[35] E. Imai, M. Horio, K. Yamagata et al., "Slower decline of glomerular filtration rate in the Japanese general population: a longitudinal 10-year follow-up study," Hypertension Research, vol. 31, no. 3, pp. 433-441, 2008. 